\title{
Editorial
}

\section{Special Issue on Quantum Optics for Fundamental Quantum Mechanics}

\author{
Marco Genovese * ${ }^{\mathbb{D}}$ and Marco Gramegna \\ INRIM, Istituto Nazionale di Ricerca Metrologica-Quantum Metrology and Nanotechnologies Division, \\ Strada delle Cacce 91, 10135 Torino, Italy; m.gramegna@inrim.it \\ * Correspondence: m.genovese@inrim.it
}

Received: 26 April 2020; Accepted: 21 May 2020; Published: 25 May 2020

\section{Introduction}

With the last turn of the century, physics has experienced the transition from the first to the second quantum revolution [1], a sweeping change that is steering the research in Quantum Mechanics towards new horizons, the most important of which is represented by the realization of the quantum computer [2], a device that nowadays represents not simply an object of desire but rather a futuristic technology in which it is worthwhile investing.

This evolution has been possible, on the one hand, thanks to great advances by scientists towards an increasingly deeper understanding of the fundamental concepts of quantum physics and, in parallel, with the dawn of quantum technology and engineering. Today, the possibility to measure, control and manipulate quantum systems at an individual level represents a basic experimental resource to be exploited in all those fields of investigation represented by quantum metrology and sensing, quantum control and quantum communication. It is definitely in relation to the development of these quantum technology tools that the possibility or not of acquiring the ability to manipulate a large number of quantum-entangled states is investigated, which is a mandatory route to reach new frontiers in quantum science.

In the depicted scenario, in which quantum mechanics is considered to have reached a stage of full maturity, fundamental questions still remain open, and in the arena of the answers that are yet have been found, quantum optics plays, as it always has, an avant-garde role: this is the reason why this Special Issue focuses mainly on this inspiring and productive research field.

\section{Quantum Optics for Fundamental Quantum Mechanics}

In light of the above, the aim of this special issue is to report theoretical and experimental works in Quantum Optics. In fact, quantum optical systems are a formidable tool for investigating the fundamental properties of physics and, specifically, of quantum mechanics. In particular, in the last 20 years, we observed exponential growth in the interest toward applications of quantum correlation, such as entanglement. This remarkable growth now encompasses a broad range of topics and we are able to witness the development of these fields, which range from foundational studies $[3,4]$ to technological aspects, covering quantum information processing [5], quantum communication [6], quantum metrology [7] and sensing [8], and quantum imaging [9].

In this Special Issue, four review papers concern some of the most interesting aspects of this field: both fundamental aspects, such a as quantum non-locality [3], uncertainty relations [10], ultra-cold bosonic atoms [11] and applicative ones, such as topological carriers of information [12].

In particular, the first manuscript [3] of the review section explores how Bell's discovery that local hidden variable theories (LHVT) cannot reproduce all the results of quantum mechanics when dealing with entangled states, which is demonstrated in the peculiarity of correlations among quantum states, 
introducing the concept of quantum non-locality, one of the most intriguing and fascinating facets of quantum mechanics, which, at the same time, remains one of the most significant problems due to the absence of its deep understanding.

In the paper [10] that celebrates the recent 90th anniversary of the emergency of the uncertainty principle, a pillar of quantum theory, the authors report the latest experimental results of uncertainty relations, quantified in terms of Shannon entropies, focusing in particular on outcomes in neutron optics and on the type of measurement uncertainties that describe the inability to obtain respective individual results from joint measurement statistics.

Superfluids, fluctuations and disorder are the topics discussed in [11], where a field-theory description of ultra-cold bosonic atoms in the presence of a disordered external potential is described: the authors investigate and review the interplay between disordered energy landscapes and fluctuations, both thermal and quantum ones, by means of functional integration techniques.

The last review paper [12] deals with topological qubits and their potential to form from the so-called winding number superpositions, where the winding number is a topologically significant quantity valuable for applications in various areas of mathematical physics, and of the consequent possibility to use these qubits as carriers of quantum information in linear optical systems, the most common realm for quantum communication.

The arguments covered in the previous section are then developed in the research articles, which consist of eight papers, offering a panorama of the present studies in quantum optics and analyzing some specific issues of the different lines of research in which this field is structured. In summary, issues concerning non-locality studies range from the application of the de Broglie-Bohm model to Gauss-Maxwell beams [13] to revisiting complementarity relations in bipartite quantum systems [14]; correlations in atoms are investigated in $[15,16]$, photon emission and statistics in $[17,18]$ and methods in quantum technologies in $[19,20]$

The first paper [13] of this section deals with a Bohmian-based approach to Gauss-Maxwell beams, a particularly appropriate and natural tool in optical problems dealing with Gaussian beams acted or manipulated by polarizers: a hydrodynamic-type extension of such a formulation is provided and discussed, complementing the notion of the electromagnetic field with that of (electromagnetic) flow or streamline. The described features confer the approach a potential interest in the analysis and description of single-photon experiments.

The complementarity relations in bipartite quantum systems are revisited in paper [14], where, by focusing on continuous-variable systems, the authors examine the influential class of EPR-like (EPR: Einstein Podolsky Rosen) states through a generalization to Gaussian states and introduce some new quantitative relations between entanglement and local interference within symmetric and asymmetric double-double-slit scenarios. In the paper, this approach is then related to ancilla-based quantum measurements and specifically to the paradigm of weak measurements.

In papers $[15,16]$, the attention moves to the investigation of correlations in atomic systems. In fact, in [15], the Electromagnetically Induced Transparency (EIT), a coherent optical nonlinear effect, is analyzed by treating the weak probe field as a perturbation to the strong coupling fields in the atomic system and using the perturbative method in a master equation: the authors show how the features of the linear response of EIT phenomena can be uniformly demonstrated, regardless of the details of atomic energy-level configuration.

On the other side, in [16], a theoretical investigation of the photon statistics in a coupled cavity system mediated by a two-level atom is shown: the authors find that there exists a photon blockade in the linear cavity for different parameter regimes, where the coupling strength between the atom and the nonlinear cavity is greater (or less) than the dissipation rate of the linear cavity. Moreover, they provide other extensions to this study to show the potential applications of a single photon source in a weakly nonlinear system.

The arguments of $[17,18]$ concern photon emission and statistics. The high-frequency electromagnetic emission from non-local wavefunctions is analyzed in [17], where it is shown that, in systems with 
non-local potentials or other kinds of non-locality, the Landauer-Büttiker formula of quantum transport leads to replacing the usual gauge-invariant current density $\mathrm{J}$ with a current $\mathrm{J}_{\text {ext }}$, which has a non-local part and coincides with the current of the extended Aharonov-Bohm electrodynamics. Peculiar properties of the analyzed scenario are analyzed by the authors to provide possible applications concerning the radiation field generated by Josephson tunneling in thick superconductor-normal-superconductor (SNS) junctions and by current flow in molecular nanodevices.

The process of second harmonic generation is addressed in a quantum frame in [18]: the analysis here starts with a perturbative approach, showing how it is possible to achieve a number of analytic results, ranging from the up-conversion probability to the statistical properties of the generated light. Moreover, the authors investigate the nonclassicality of some benchmark states by inspecting the corresponding autocorrelation function.

Finally, this Special Issue concludes with methods in quantum technologies [19,20], a very promising and vivid field that nowadays centralizes the great efforts of the quantum optics community.

The study reported in [19] articulates the context of multiparameter quantum estimation theory: the authors investigate the construction of linear schemes in order to infer two classical parameters that are encoded in the quadratures of two quantum coherent states. The optimality of the scheme, built on two-phase conjugated coherent states, is proven with the saturation of the quantum Cramér- Rao bound under global energy constraints. The study is then extended on n-mode schemes that can be used to encode $\mathrm{n}$ classical parameters into $\mathrm{n}$ quantum coherent states, and then estimate all parameters optimally and simultaneously.

In conclusion, paper [20] focuses on Quantum Key Distribution (QKD) Networks, that actually are an important support for quantum communication. QKD Networks have achieved a relatively mature level of development and, consequently, are facing greater requirements in terms of their implementation capabilities, thus requiring an effective management plane to control and coordinate the QKD resources. The presented study reports how, as a promising technology, software-defined networking (SDN) can separate the control and management of QKD networks from the actual forwarding of the quantum keys.

Funding: This research was supported by grant number (FQXi FFF Grant number FQXi-RFP-1812) from the Foundational Questions Institute and Fetzer Franklin Fund, a donor advised fund of Silicon Valley Community Foundation. This work has received funding from PATHOS EU H2020 FET-OPEN grant no. 828946, and from the European Union Horizon 2020 and the EMPIR Participating States in the context of the project EMPIR-17FUN01 'BeCOMe'.

Acknowledgments: As Guest Editors of this Special Issue, we would like to thank all of the authors and anonymous peer reviewers for their valuable contributions. Undoubtedly, this issue would not be possible without the excellent and tireless work done by the MDPI management and staff, to whom we convey our most sincere thanks, with a special thanks to Daria Shi, Managing Editor at the MDPI Branch Office, Beijing.

Conflicts of Interest: The authors declare no conflict of interest.

\section{References}

1. Dowling, J.P.; Milburn, G.J. Quantum technology: The second quantum revolution. Philos. Trans. R. Soc. A 2003, 361, 1655-1674. [CrossRef] [PubMed]

2. Arute, F.; Arya, K.; Babbush, R.; Bacon, D.; Bardin, J.C.; Barends, R.; Biswas, R.; Boixo, S.; Brandao, F.G.S.L.; Buell, D.A.; et al. Quantum supremacy using a programmable superconducting processor. Nature 2019, 574, 505-510. [CrossRef] [PubMed]

3. Genovese, M.; Gramegna, M. Quantum correlations and quantum non-locality: A review and a few new ideas. Appl. Sci. 2019, 9, 5406. [CrossRef]

4. Genovese, M. Interpretations of quantum mechanics and measurement problem. Adv. Sci. Lett. 2010, 3, 249-258. [CrossRef]

5. Flamini, F.; Spagnolo, N.; Sciarrino, F. Photonic quantum information processing: A review. Rep. Prog. Phys. 2019, 82, 016001. [CrossRef] [PubMed] 
6. Pirandola, S.; Andersen, U.L.; Banchi, L.; Berta, M.; Bunandar, D.; Colbeck, R.; Englund, D.; Gehring, T.; Lupo, C.; Ottaviani, C.; et al. Advances in quantum cryptography. arXiv 2019, arXiv:1906.01645. [CrossRef]

7. Giovannetti, V.; Lloyd, S.; Maccone, L. Advances in quantum metrology. Nat. Photonics 2011, 5, $222-229$. [CrossRef]

8. Degen, C.L.; Reinhard, F.; Cappellaro, P. Quantum sensing. Rev. Mod. Phys. 2017, 89, 035002. [CrossRef]

9. Genovese, M. Real applications of quantum imaging. J. Opt. 2016, 18, 073002. [CrossRef]

10. Demirel, B.; Sponar, S.; Hasegawa, Y. Measurements of entropic uncertainty relations in neutron optics. Appl. Sci. 2020, 10, 1087. [CrossRef]

11. Cappellaro, A.; Salasnich, L. Superfluids, fluctuations and disorder. Appl. Sci. 2019, 9, 1498. [CrossRef]

12. Jaeger, G.; Simon, D.; Sergienko, A. Topological qubits as carriers of quantum information in optics. Appl. Sci. 2019, 9, 575. [CrossRef]

13. Sanz, A.S.; Davidovic, M.D.; Božic, M. Bohmian-Based approach to Gauss-Maxwell beams. Appl. Sci. 2020, 10, 1808. [CrossRef]

14. Peled, B.; Te'eni, A.; Georgiev, D.; Cohen, E.; Carmi, A. Double slit with an Einstein-Podolsky-Rosen pair. Appl. Sci. 2020, 10, 792. [CrossRef]

15. Wang, X. A perturbative view from the master equation: Electromagnetically induced transparency revisited. Appl. Sci. 2019, 9, 4512. [CrossRef]

16. Li, M.; Chen, A. A photon blockade in a coupled cavity system mediated by an atom. Appl. Sci. 2019, 9, 980. [CrossRef]

17. Modanese, G. High-Frequency electromagnetic emission from non-local wavefunctions. Appl. Sci. 2019, 9, 1982. [CrossRef]

18. Chesi, G.; Wauters, M.; Fasola, N.; Allevi, A.; Bondani, M. Second harmonic revisited: An analytic quantum approach. Appl. Sci. 2019, 9, 1690. [CrossRef]

19. Arnhem, M.; Karpov, E.; Cerf, N. Optimal estimation of parameters encoded in quantum coherent state quadratures. Appl. Sci. 2019, 9, 4264. [CrossRef]

20. Wang, H.; Zhao, Y.; Nag, A. Quantum-Key-Distribution (QKD) networks enabled by Software-Defined Networks (SDN). Appl. Sci. 2019, 9, 2081. [CrossRef]

(C) 2020 by the authors. Licensee MDPI, Basel, Switzerland. This article is an open access article distributed under the terms and conditions of the Creative Commons Attribution (CC BY) license (http://creativecommons.org/licenses/by/4.0/). 